\title{
A model for cortical remapping and structural plasticity following focal retinal lesions Markus Butz ${ }^{* 1,2}$, Florentin Wörgötter ${ }^{2}$ and Arjen van Ooyen ${ }^{1}$
}

Address: ${ }^{1}$ Dept. for Integrative Neurophysiology, VU University, Amsterdam, 1081NL, The Netherlands and ${ }^{2}$ Bernstein Center for Computational Neuroscience, University of Göttingen, 37073 Göttingen, Germany

Email: Markus Butz* - mbutz@cncr.vu.nl

* Corresponding author

from Eighteenth Annual Computational Neuroscience Meeting: CNS*2009

Berlin, Germany. 18-23 July 2009

Published: 13 July 2009

BMC Neuroscience 2009, I0(SuppI I):P206 doi:I0.II86/I47I-2202-I0-SI-P206

This abstract is available from: http://www.biomedcentral.com/I47I-2202/I0/SI/P206

(c) 2009 Butz et al; licensee BioMed Central Ltd.

It is still debatable to what extent structural plasticity in terms of synaptic rewiring is the cause for lesion-induced or experience-dependent cortical remapping [1]. Recent two-photon laser imaging studies demonstrate that synaptic rewiring is persistent in the adult brain and is dramatically increased following brain lesions or after a loss of sensory input (deafferentation). We use a recurrent neural network model [2] as a vehicle to study structural plasticity; to study the time course of synaptic rewiring following a lesion, we represent the synapse as consisting of axonal (terminals/varicosities) and dendritic elements (spines). Independent development of both pre- and postsynaptic elements allows for modelling synapse formation, pruning and synaptic turnover as distinct processes. Model neurons increase and decrease axonal and dendritic elements in an activity-dependent fashion. Hence, synaptic rewiring is subject to shifts in the excitation-inhibition equilibrium. We apply this model to recent experimental data from Keck et al. [3] on cortical remapping following focal retinal lesion. The model could also be applied to somatosensory deafferentation. In this study we demonstrate that maintaining network homeostatis and rebalancing deafferented neurons by synaptic rewiring can result in post-lesion cortical remapping. Thus, the model bridges the gap between activitydependent morphological changes on the neuronal level and a changing connectivity of cortical maps on an anatomical level. These theoretical results could have large consequences for neurological rehabilitation after stroke.

\section{References}

I. Butz M, Wörgötter F, van Ooyen A: Activity-dependent structural plasticity. Brain Res Rev 2009 in press.

2. Butz M, Teuchert-Noodt G, Grafen K, van Ooyen A: Inverse relationship between adult hippocampal cell proliferation and synaptic rewiring in the dentate gyrus. Hippocampus 2008 , 18:879-898.

3. Keck T, Mrsic-Flogel TD, Vaz Afonso M, Eysel UT, Bonhoeffer T, Hübener M: Massive restructuring of neuronal circuits during functional reorganization of adult visual cortex. Nat Neurosci 2008, II:I162-I167. 